\title{
On Unique Games with Negative Weights
}

\author{
Peng Cui ${ }^{1}$ \\ Key Laboratory of Data Engineering and Knowledge Engineering, MOE, School of Information \\ Resource Management, Renmin University of China, Beijing 100872, P. R. China. \\ cuipeng@ruc.edu.cn
}

\begin{abstract}
In this paper, the author defines Generalized Unique Game Problem (GUGP), where weights of the edges are allowed to be negative. Two special types of GUGP are illuminated, GUGP-NWA, where the weights of all edges are negative, and GUGP-PWT $(\rho)$, where the total weight of all edges are positive and the negative-positive ratio is at most $\rho$. The author investigates the counterpart of the Unique Game Conjecture on GUGP-PWT $(\rho)$. The author shows that Unique Game Conjecture on GUGP-PWT(1) holds true, and Unique Game Conjecture on GUGP-PWT(1/2) holds true, if the 2-to-1 Conjecture holds true. The author poses an open problem whether Unique Game Conjecture holds true on GUGP$\operatorname{PWT}(\rho)$ with $0<\rho<1$.
\end{abstract}

\section{Introduction}

The Unique Game Conjecture (UGC) is put forward by Khot on STOC 2002 as a powerful tool to prove lower bound of inapproximabilty for combinatorial optimization problems [6]. It has been shown by researchers a positive resolution of this conjecture would imply improved even best possible hardness results for many famous problems, to name a few, Max Cut, Vertex Cover, Multicut, Min 2CNF Deletion, making an important challenge to prove or refute the conjecture.

Some variations of UGC have been mentioned. Rao proves a strong parallel repetition theorem which shows Weak Unique Game Conjecture is equivalent to UGC[10]. Khot et al. show that Unique Game Conjecture on Max 2LIN(q) is equivalent to UGC[7]. Khot poses the d-to-1 Conjectures in his original paper for $d \geq 2[6]$. O'Donell et al. show a tight hardness for approximating satisfiable constraint satisfaction problem on 3 Boolean variables assuming the d-to-1 Conjecture for any fixed $d[8]$. Dinur et al. use the 2-to-2 Conjecture to derive the hardness results of Approximate Coloring Problem, and prove that the 2-to-1 Conjecture implies their 2-to-2 Conjecture[2]. Guruswami et al. use the 2-to-1 Conjecture to derive the hardness result of Maximum k-Colorable Subgraph Problem[4]. It is unknown whether UGC implies any of the d-to-1 Conjectures, or vice versa.

Recently, the authors of [1] designed a subexponential time algorithm for Unique Games Problem (UGP), which challenges the position of UGC as a tool to prove lower bound of inapproximabilty. While their results stop short of refuting UGC, they do suggest that UGP is significantly easier than NP-hard problems. On the other side, the authors of [9] determined a new point of $(c, s)$-approximation NP-hardness of UGP, 
compared to [5], and their new result, together with the result of [3], determines the two-dimensional region of all known $(c, s)$-approximation NP-hardness of UGP.

In this paper, the author defines Generalized Unique Game Problem (GUGP), where weights of the edges are allowed to be negative. Two special types of GUGP are illuminated, GUGP-NWA, where the weights of all edges are negative, and GUGP-PWT $(\rho)$, where the total weight of all edges are positive and the negative-positive ratio is at most $\rho$. GUGP-PWT $(\rho)$ over $1 \geq \rho \geq 0$ makes a possible phase transition from a 2-Prover 1-Round Game Problem with $(1-\zeta, \delta)$-approximation NP-hardness to UGP. The author shows that UGC on GUGP-PWT(1) holds true, UGC on GUGP-PWT(1/2) holds true, if the 2-to-1 Conjecture holds true, and the $(1-\zeta, \delta)$-approximation NP-hardness of GUGP-PWT $(\rho)$ possesses the compactness property when $\rho \rightarrow 0$.

Section 2 demonstrates some definitions. The author shows the main results for GUGP-NWA and GUGP-PWT $(\rho)$ in Section 3. Section 4 is some discussions.

\section{Preliminaries}

In 2-Prover 1-Round Game Problem (2PIR), we are given a bipartite graph $G=(V, W ; E)$, with each edge $e$ having a weight $w_{e} \in \mathbb{Q}^{+}$. We are also given two sets of labels, $k_{1}$ and $k_{2}$, which we identify with $\left[k_{1}\right]=\left\{1, \cdots, k_{1}\right\}$ and $\left[k_{2}\right]=\left\{1, \cdots, k_{2}\right\}$. Each edge $e=(u, v)$ in the graph is equipped with a relation $R_{e} \subseteq\left[k_{1}\right] \times\left[k_{2}\right]$. The solution of the problem is a labeling $f_{1}: V \rightarrow\left[k_{1}\right]$ and $f_{2}: W \rightarrow\left[k_{2}\right]$ which assigns a label to each vertex of $G$. An edge $e=(u, v)$ is said to be satisfied under $f_{1}$ and $f_{2}$ if $\left(f_{1}(u), f_{2}(v)\right) \in R_{e}$, else is said to be unsatisfied. The object of the problem is to find a labeling maximizing the total weight of the satisfied edges. The value of the instance, $\operatorname{Val}(G)$, is defined as the maximum total weight of the satisfied edges divided by the total weight of all edges.

Unique Game Problem (UGP) can be viewed as a special type of 2P1R. In UGP, we are given a graph $G=(V, E)$, a weight function $w_{e} \in \mathbb{Q}^{+}$for $e \in E$, and a set of labels, [k]. Each edge $e=(u, v)$ in the graph is equipped with a permutation $\pi_{e}:[k] \rightarrow[k]$. The solution of the problem is a labeling $f: V \rightarrow[k]$ which assigns a label to each vertex of $G$. An edge $e=(u, v)$ is said to be satisfied under $f$ if $\pi_{e}(f(u))=f(v)$, else is said to be unsatisfied. Note that we allow $G$ is a graph with parallel edges, i.e., there exist more than one edge between two vertices.

It is possible to define two optimization problems in this situation. In Max UGP, the value of the instance is defined as the maximum total weight of the satisfied edges divided by the total weight of all edges. In Min UGP, the value of the instance is defined as the minimum total weight of the unsatisfied edges divided by the total weight of all edges.

Khot initiates much of the interest in the following conjecture by showing that many hardness results stem from it. It basically states that it is NP-hard to distinguish whether many or only few edges are satisfied.

Conjecture 1. ([6] Unique Game Conjecture in Max UGP Form) For every $\zeta, \delta>0$, there is a $k=k(\zeta, \delta)$ such that given an instance $G$ of Max UGP with $k$ labels it is $N P$-hard to distinguish whether $\operatorname{Val}(G)>1-\zeta$ or $\operatorname{Val}(G)<\delta$. 
The conjecture can be restated in Min UGP form, and the two conjectures are equivalent.

Conjecture 2. (Unique Game Conjecture in Min UGP Form) For every $\zeta, \delta>0$, there is a $k=k(\zeta, \delta)$ such that given an instance $G$ of Min UGP with $k$ labels it is $N P$-hard to distinguish whether $\operatorname{Val}(G)<\zeta$ or $\operatorname{Val}(G)>1-\delta$.

The $(c, s)$-approximation NP-hardness of Max UGP is defined as: for some fixed $0<s<c<1$, there is a $k$ such that given an instance $G$ of Max UGP with $k$ labels it is NP-hard to distinguish whether $\operatorname{Val}(G) \geq c$ or $\operatorname{Val}(G)<s+\varepsilon$ for any $\varepsilon>0$.

2-to-1 Game and 2-to-2 Game are two special types of 2P1R. In 2-to-1 Game, we are given a bipartite graph $G=(V, W ; E)$, with each edge $e$ having a weight $w_{e} \in \mathbb{Q}^{+}$. We are also given two sets of labels, [2k] for $V$ and $[k]$ for $W$. Each edge $e=(u, v)$ in the graph is equipped with a 2-to-1 projection. A projection $\sigma:[2 k] \rightarrow[k]$ is said to be a 2-to-1 projection if for each element $j \in[k]$ we have $\left|\sigma^{-1}(j)\right|=2$. The value of the instance of 2-to-1 Game, $\operatorname{Val}(G)$, is defined as the maximum total weight of the satisfied edges divided by the total weight of all edges.

In 2-to-2 Game, we are given a graph $G=(V, E)$, a weight function $w_{e} \in \mathbb{Q}^{+}$for $e \in E$, and a set of labels, $[k]$. Each edge $e=(u, v)$ in the graph is equipped with a 2-to-2 relation. A relation $R \subseteq[2 k] \times[2 k]$ is said to be a 2-to-2 relation if there are two permutations $\pi_{u}, \pi_{v}:[2 k] \rightarrow[2 k]$ such that $(i, j) \in R$ iff $\left(\pi_{u}(i), \pi_{v}(j)\right) \in T$ where

$$
T:=\bigcup_{l=1}^{k}\{(2 l-1,2 l-1),(2 l-1,2 l),(2 l, 2 l-1),(2 l, 2 l)\} .
$$

The value of the instance of 2-to-2 Game, $\operatorname{Val}(G)$, is defined as the maximum total weight of the satisfied edges divided by the total weight of all edges.

The author lists the 2-to-1 Conjecture and the 2-to-2 Conjecture in their maximization forms. Note that the latter is somewhat different from that in [2]. It can be proved that the 2-to-1 Conjecture implies the 2-to-2 Conjecture along the line of [2].

Conjecture 3. (2-to-1 Conjecture) For every $\delta>0$, there is a $k=k(\delta)$ such that given an instance $G$ of 2-to-1 Game with the label sets [2k] and $[k]$ it is NP-hard to distinguish whether $\operatorname{Val}(G)=1$ or $\operatorname{Val}(G)<\delta$.

Conjecture 4. (2-to-2 Conjecture) For every $\delta>0$, there is a $k=k(\delta)$ such that given an instance $G$ of 2-to-2 Game with the label set $[2 k]$ it is NP-hard to distinguish whether $\operatorname{Val}(G)=1$ or $\operatorname{Val}(G)<\delta$.

In this paper, the author defines Generalized Unique Game Problem (GUGP), where the weights of edges are allowed to be negative. In GUGP, we are given a graph $G=$ $(V, E)$, possibly having parallel edges, a weight function $w_{e} \in \mathbb{Q}$ for $e \in E$, and a set of labels, $[k]$. Each edge $e=(u, v)$ in the graph is equipped with a permutation $\pi_{e}:[k] \rightarrow[k]$. The solution of GUGP is a labeling $f: V \rightarrow[k]$ which assigns a label to each vertex of $G$. The goal of the problem is to maximize the total weight of the 
satisfied edges. Note that $w_{e}$ could be positive or negative. The author assumes there is no edge with zero weight for sake of clearance.

Let $W_{G}^{+}$be the total of the positive weights of all edges, $W_{G}^{-}$be the total of the negative weights of all edges, and $\Sigma_{G}=W_{G}^{+}+W_{G}^{-}$be the total weight of all edges. The author calls $r_{G}=\left|W_{G}^{-}\right| / W_{G}^{+}$the negative-positive ratio of the instance.

GUGP-NWA and GUGP-PWT are two special types of GUGP. In GUGP-NWA, the weight of all edges are negative. In GUGP-PWT, the total weight of all edges is positive. It is possible to define two optimization problems for GUGP-NWA and for GUGP-PWT.

In Max GUGP-NWA, we seek to minimize the total weight of the unsatisfied edges, i.e. to maximize the absolute value of the total weight of the unsatisfied edges. The value of Max GUGP-NWA is defined as the maximum absolute value of the total weight of the unsatisfied edges divided by $\left|W_{G}^{-}\right|$. In Min GUGP-NWA, we seek to maximize the total weight of the satisfied edges, i.e. to minimize the absolute value of the total weight of the satisfied edges. The value of Min GUGP-NWA is defined as the minimum absolute value of the total weight of the satisfied edges divided by $\left|W_{G}^{-}\right|$.

In Max GUGP-PWT, we seek to maximize the total weight of the satisfied edges. The value of Max GUGP-PWT is defined as the maximum total weight of the satisfied edges divided by $\Sigma_{G}$. In Min GUGP-PWT, we seek to minimize the total weight of the unsatisfied edges. The value of Min GUGP-PWT is defined as the minimum total weight of the unsatisfied edges divided by $\Sigma_{G}$. In an instance $G$ of Min GUGP-PWT, let $W_{G}(f)$ be the total weight of the unsatisfied edges under labeling $f$, and let the optimal labeling be $f^{*}$. The value of the instance is $\operatorname{Val}(G)=W_{G}\left(f^{*}\right) / \Sigma_{G}$.

The author reminds the reader that the value of Max GUGP-PWT could be more than 1 and and the value of Min GUGP-PWT could be less than 0 .

The author defines Max/Min GUGP-PWT( $\rho)$ as the subproblem of Max/Min GUGPPWT where the negative-positive ratio of the instances is upper bounded by $\rho$, where $\rho$ is a constant independent from $k$. Since the negative-positive ratio is always less than 1 , we set the range of $\rho$ to be $0 \leq \rho \leq 1$. Note that Max/Min GUGP-PWT(0) is simply Max/Min UGP.

The author gives the two equivalent counterparts of the Unique Game Conjecture on Max GUGP-PWT $(\rho)$ and Min GUGP-PWT $(\rho)$ ) as follows:

Conjecture 5. (Unique Game Conjecture on Max GUGP-PWT $(\rho)$ ) For every $\zeta, \delta>$ 0 , there is a $k=k(\zeta, \delta)$ such that given an instance of Max GUGP-PWT( $\rho)$ with $k$ labels it is NP-hard to distinguish whether $\operatorname{Val}(G)>1-\zeta$ or $\operatorname{Val}(G)<\delta$.

Conjecture 6. (Unique Game Conjecture on Min GUGP-PWT $(\rho)$ ) For every $\zeta, \delta>$ 0 , there is a $k=k(\zeta, \delta)$ such that given an instance of Min GUGP-PWT $(\rho)$ with $k$ labels it is $N P$-hard to distinguish whether $\operatorname{Val}(G)<\zeta$ or $\operatorname{Val}(G)>1-\delta$.

The conjectures states it is NP-hard to distinguish the following two cases: there is a labeling under which the absolute value of the total of the negative weight of the unsatisfied edges is almost no less than the total of the positive weight of the unsatisfied edges; under any labeling the absolute value of the total of the negative weight of the 
satisfied edges is almost no less than the total of the positive weight of the satisfied edges.

\section{Main Results}

\subsection{GUGP-NWA}

Max GUGP-NWA can be restated as the following 2P1R. We are given a graph $G=$ $(V, E)$, a weight function $w_{e} \in \mathbb{Q}^{+}$, and a set of labels, $[k]$. Each edge $e=(u, v)$ in the graph is equipped with a relation $\bar{\pi}_{e}=[k] \times[k]-\pi_{e}$, where $\pi_{e}:[k] \rightarrow[k]$ is a permutation. The solution of the problem is a labeling $f: V \rightarrow[k]$ which assigns a label to each vertex of $G$. An edge $e=(u, v)$ is said to be satisfied under $f$ if $(f(u), f(v)) \in \bar{\pi}_{e}$. The value of the instance is defined as the total weight of the satisfied edges divided by the total weight of all edges.

Since a random labeling satisfies an expectation of $1-1 / k$ fraction of the total weight of all edges, GUGP-NWA cannot have a large gap. We can prove that it is NPhard to approximate Min GUGP-NWA within any poly $(k)$, by the similar arguments in the following theorem. The author omits the full proof for clarity of the paper.

Theorem 1. It is NP-hard to approximate Min GUGP-NWA with the label set $[n]$ within any $\operatorname{poly}(n)$.

Proof. Min GUGP-NWA can be restated as: In the situation of UGP, the goal is to find minimum fraction of the total weight of the satisfied edges. The author constructs an approximation ratio preservation reduction from TSP to the above problem.

Given an instance of TSP problem $G=(V, E)$, where each edge of $E$ has a weight $w_{e} \in \mathbb{Q}^{+}$. Denote $n:=|V|$. The instance of the restated form of Min GUGP-NWA is a graph $G^{\prime}=G^{\prime}\left(V, E^{\prime}\right)$, with each edge $e^{\prime} \in E^{\prime}$ having a weight $w^{\prime}\left(e^{\prime}\right)$, and with the labeling set $[n]$. For each edge $e=(u, v) \in E$, there are three parallel edges $e^{=}, e^{+}$and $e^{-}$between $u$ and $v$ in $E^{\prime} . e^{=}$has weight $M$ and equipped with permutation $\pi^{=}=\{(1,1),(2,2), \cdots,(n, n)\}$. Let $M=n \cdot \operatorname{Max}(w)$, where $\operatorname{Max}(w)$ is the maximum weight of all edges in $G . e^{+}$has weight $\left.w_{(} e\right)$ and equipped with permutation $\pi^{+}=\{(1,2),(2,3), \cdots,(n, 1)\} . e^{-}$has weight $\left.w_{(} e\right)$ and equipped with permutation $\pi^{-}=\{(1, n),(2,1), \cdots,(n, n-1)\}$.

Given a solution of TSP problem, a Hamiltonian cycle $C$, we can assign label 1 to $n$ to vertices of $C$ along $C$ in $G^{\prime}$, and the total weight of satisfied edges in $G^{\prime}$ is exactly the total weight of edges on $C$ in $G$.

In the other direction, given a labeling $f$ of $G^{\prime}$, if there are two vertices assigned with the same label, the total weight of the satisfied edges is at least $M$. Otherwise all vertices are assigned with label from 1 to $n$ respectively, let $u_{i}$ be the vertices assigned label $i$ for $1 \leq i \leq n$, and $e_{i}^{+} \in E^{\prime}$ be the edge between $u_{i}$ and $u_{i} \bmod n+1$ equipped with permutation $\pi^{+}$. The total weight of the satisfied edges is equal to $\sum_{1 \leq i \leq n} w^{\prime}\left(e_{i}^{+}\right)$. Let $C$ be the Hamiltonian cycle of $G$ which consists of vertices from $u_{1}$ to $u_{n}$, then the total weight of $C$ in $G$ is exactly the total weight of satisfied edges under $f$ in $G^{\prime}$. 


\subsection{GUGP-PWT $(\rho)$}

By [3], for any constant $C>0$, there is a $\varepsilon>0$, such that $(C \varepsilon, \varepsilon)$-approximating UGP is NP-hard. Note that $C \varepsilon \rightarrow 0$, when $\varepsilon \rightarrow 0$. We get an instance of GUGP-PWT $(1-C \varepsilon)$, by supplementing the graph in the instance of UGP with two new vertices and $k$ parallel edges between the two vertices, each of which has the negative weight $-\frac{1-C \varepsilon}{k}$ and is equipped with one of the permutations $\pi_{i}: j \mapsto(j+i-2) \bmod k+1$ for $1 \leq i \leq k$.

For every $\zeta, \delta>0$, we can determine $C$ such that $\frac{1}{C}<\delta$, and the size of the label set of the instance, $k$, satisfies $\frac{1}{k C \varepsilon}<\zeta$. Then for this instance of Max GUGP-PWT( $\left.1-C \varepsilon\right)$ with $k$ labels, $G$, it is NP-hard to distinguish whether $\operatorname{Val}(G)>1-\zeta$ or $\operatorname{Val}(G)<\delta$. Therefore, Conjecture 6 holds true for $\rho=1$.

Conjecture 4 implies the $(1 / 2,0)$-approximation NP-hardness of Max UGP by the following reduction. Write each edge in an instance of the 2-to-2 game into two parallel edges, each of which has the same weight as the original edge and is equipped with one of the two disjoint permutations extracted from the original 2-to-2 relation. Thus Conjecture 4 implies Conjecture 6 holds true for $\rho=1 / 2$ by the similar technique in the first paragraph of this subsection. Since Conjecture 3 implies Conjecture 4, Conjecture 6 holds true for $\rho=1 / 2$ if Conjecture 3 holds true.

Finally, we establish a connection from Conjecture 6 to the Unique Game Conjecture by the following theorem.

Theorem 2. If Conjecture 6 holds true for any $\rho>0$, Conjecture 2 holds true.

Proof. Suppose Conjecture 2 holds false, then for some $\zeta, \delta>0$, for any label size $k$, we can decide in polynomial time whether an instance of Min UGP with $k$ labels has a value more than $1-\delta$ or less than $\zeta$. The author claims Conjecture 6 for $\rho=\min (\zeta, \delta) / 2$ holds false.

Given an instance $G=(V, E)$ of Min GUGP-PWT $(\rho)$, we construct an instance $G^{\prime}=\left(V, E^{\prime}\right)$ of Min UGP as follows. Let $E^{\prime}$ be the set of the edges in $E$ with positive weights. Let $f^{*}$ be the optimal labeling of $G$, and $f^{\prime}$ be the optimal labeling of $G^{\prime}$. Then $\operatorname{Val}\left(G^{\prime}\right)=W_{G^{\prime}}\left(f^{\prime}\right) / W_{G}^{+}$and $\operatorname{Val}(G)=W_{G}\left(f^{*}\right) / \Sigma_{G}$.

Since $W_{G^{\prime}}\left(f^{\prime}\right) \geq W_{G}\left(f^{\prime}\right) \geq W_{G}\left(f^{*}\right)$ and $\Sigma_{G} / W_{G}^{+} \geq 1-\rho, \operatorname{Val}\left(G^{\prime}\right) \geq(1-\rho) \operatorname{Val}(G)$.

By the definition of $E^{\prime}, W_{G}\left(f^{*}\right) \geq W_{G^{\prime}}\left(f^{*}\right)-\rho W_{G}^{+}$. We have $\operatorname{Val}(G) \Sigma_{G}=W_{G}\left(f^{*}\right) \geq$ $W_{G^{\prime}}\left(f^{*}\right)-\rho W_{G}^{+} \geq W_{G^{\prime}}\left(f^{\prime}\right)-\rho W_{G}^{+}=\left(\operatorname{Val}\left(G^{\prime}\right)-\rho\right) W_{G}^{+}$. Therefore, $\operatorname{Val}\left(G^{\prime}\right) \leq \operatorname{Val}(G)+\rho$.

If $\operatorname{Val}(G)<\zeta / 2$, then $\operatorname{Val}\left(G^{\prime}\right)<\zeta$. If $\operatorname{Val}(G)>1-\delta / 2$, then $\operatorname{Val}\left(G^{\prime}\right)>1-\delta$. Thus we can decide in polynomial time whether the instance of Min GUGP-PWT $(\rho)$ has a value more than $1-\delta / 2$ or less than $\zeta / 2$.

\section{Discussions}

It leaves as an open problem whether Unique Game Conjecture holds true on GUGP$\operatorname{PWT}(\rho)$ for $0<\rho<1$. The author makes a reasonable and rather bold conjecture: If Conjecture 6 holds true for some $0<\rho<1$, it holds true for any $0<\rho<1$, which would lead to the corollary that the 2-to-1 Conjecture implies the Unique Game Conjecture. To confirm our conjecture, it would be critical to seek techniques to derive the $(1-\zeta, \delta)$-approximation NP-hardness on smaller $\rho$ by the $(1-\zeta, \delta)$-approximation NP-hardness on larger $\rho$. 


\section{References}

1. S. Arora, B. Barak, D. Steurer, Subexponential algorithm for Unique Games and related problems, in: Proc. 52th Annual IEEE Symposium on Foundations of Computer Science, 2010, pp. 563-572

2. I. Dinur, E. Mossel, O. Regev, Conditional hardness for approximate coloring, in: Proc. 38th Annual ACM Symposium on Theory of Computing, 2006, pp. 344-353

3. U. Feige, D. Reichman, On system of linear equations with two variables per equation, in: Proc. ARRROX-RANDOM, 2004, pp. 117-127

4. V. Guruswami, A. K. Sinop, Improved inapproximability results for Maximum k-Colorable Subgraph, in: Proc. ARRROX-RANDOM, 2009, pp. 163-176

5. J. Håstad, Some optimal inapproximability results, Journal of the ACM 48 (2001) 798-859

6. S. Khot, On the power of unique 2-prover 1-round games, in: Proc. 34th Annual ACM Symposium on Theory of Computing, 2002, pp. 767-775

7. S. Khot, G. Kindler, E. Mossel, R. O'Donnell, Optimal inapproximability results for MaxCut and other 2-variable CSPs? in: Proc. 45th Annual IEEE Symposium on Foundations of Computer Science, 2004, pp. 146-154

8. R. O'Donnell, Y. Wu, Conditional hardness for satisfiable CSPs, in: Proc. 39th Annual ACM Symposium on Theory of Computing, 2009, pp. 493-502

9. R. O'Dornell, J. Wright, A new point of NP-hardness for Unique games, in: Proc. 44th Annual ACM Symposium on Theory of Computing, 2012, pp. 289-306

10. A. Rao, Parallel repitition in projection games and a concentration bound, In: Proc. 38th Annual ACM Symposium on Theory of Computing, 2008, pp. 1-10 